\title{
An Optimized Sensing and Detection of Cognitive Radio Network using Monte Carlo Simulation
}

\author{
Abhinav Shukla \\ M.Tech (DC) Scholar \\ NIIST, Bhopal
}

\author{
Puran Gour \\ Professor, Dept. of EC \\ NIIST, Bhopal
}

\begin{abstract}
The Cognitive Radio Network is intelligent network, which has the capability to efficiently utilize the available spectrum using various spectrum-sensing techniques, in addition with the intelligent energy consumption and bandwidth allocation. In this paper we are simulating the cognitive radio network using Monte-Carlo simulation model. The proposed system is tested under Additive White Gaussian noise (AWGN) channel and Rayleigh Fading Channel environment. During simulation the probability of detection $(\mathrm{Pd})$ is calculated for given signal to noise ratio (SNR) and false alarm rate (Pf). To enhance the system performance median filter is implemented which significantly enhances the performance of detection probability for given SNR and Pf.
\end{abstract}

\section{Keywords}

Probability of Detection(Pd), False Alarm Rate(Pf), SNR, Monte-Carlo Simulation and Median Filtering.

\section{INTRODUCTION}

The term "cognitive radio" defines the wireless systems that can sense, be aware of, learn, and adapt to the surrounding environment according to inner and outer stimuli. Overall cognition cycle[3] can be seen as an instance of artificial intelligence, since it encompasses observing, learning, reasoning, and adaptation. The adaptation itself in the cognition cycle is a complex problem, because cognitive radio needs to take into account several inputs at the same time including its own past observations as a result of learning property. Although the adaptation of wireless networks[5] is not a new conception, the earlier standards and technologies strive to obtain an adaptive wireless communication network from a narrower perspective (commonly focused on a singlelayer adaptation with a single objective function) as compared to that of cognitive radio, which considers a global adaptation that includes multiple layers and goal functions.

For numerous researchers and technocrats, the cognitive radio idea is not limited to a single intelligent radio, but it includes networking functionalities. Cognitive networks $(\mathrm{CN})[2]$ can be defined as intelligent networks that can automatically sense the environment (individually and collaboratively) and current network conditions, and adapt the communication parameters accordingly. As comparing to the cognitive radio and cognitive networks definition, it can be seen that the definitions are analogous, except cognitive networks have further broader perspective that also include all the network elements. The cognitive networks are expected to shape the future wireless networks with important applications in dynamic spectrum access, coexistence and interoperability of different wireless networks. Amongst the special features of cognitive networks, the leading ones are advanced interference management strategies, safe and secure wireless access methodologies, efficient use of wireless resources and excellent Quality of Service[1]. In spite of all these great features and possibilities, being a new concept, the cognitive radio network poses many new technical challenges. As it is described in the successive sections, these networks have requirements in dynamic spectrum management, complexity and size, power and hardware efficiency, spectrum sensing and interference identification, environment awareness, new distributed algorithm design[6], distributed spectrum measurements, Quality of Service guarantees, user awareness, location awareness and security

\section{SYSTEM MODEL \& COGNITIVE RADIO NETWORKS ARCHITECTURE}

Here a cognitive radio network containing $\mathrm{N}$ CRs is considered, one PU, and an FC. It has assumed that each of the FC and PU contains a single antenna and each CR contains $\mathrm{M}$ antennas and there are two hypotheses $\mathrm{H} 0$ and $\mathrm{H} 1$ consequent to the signal received in the $\mathrm{i}^{-} \mathrm{th}$ antenna at each CR,

Where the antenna index denotes the signal transmitted by the $\mathrm{PU}$ at time instant $t$ with energy is circularly symmetrical

complex AWGN, and all are independent and identically distributed complex normal circularly symmetrical channel gains implying Rayleigh fading. It is assumed that the CRs do not have any information about the channels of the PU-CR links. Further, it is assumed that each CR contains the improved energy detector [2]; the statistic at the antenna for deciding the presence or absence of the PU is given by

where we have dropped the time index t for simplicity. It can be seen from (2) that reduces to the statistic corresponding to the conventional energy detector [1]. For above the discussed set-up, cooperative spectrum sensing is performed as follows:

a) Each $\mathrm{CR}$ calculates decision statistic given in equation (2) for all antennas and uses selection combining for taking a binary decision of a spectrum hole.

b) The binary decision of every $\mathrm{CR}$ is sent to the FC over an imperfect reporting channel.

c) The FC applies the ' $\mathrm{OR}$ ' rule to the binary decisions received from all CRs and takes a final decision on whether the PU is present or not. 


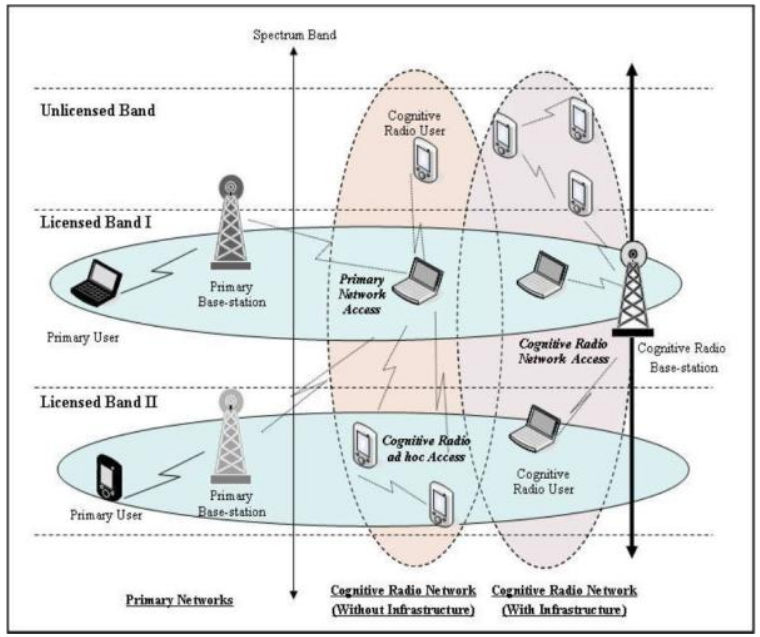

Fig. 1. Shows Cognitive radio network architecture [4]

Current wireless network environment employs heterogeneity in terms of both spectrum policy and communication technologies. Therefore, a clear description of the cognitive radio network architecture is crucial for development of communication protocols[7]. The components of the cognitive radio network architecture, as shown in Fig. 1, can be classified in two groups as the primary network and cognitive network $(\mathrm{CN})$. Primary network is said to as the legacy network that has an exclusive right to a certain spectrum band. The cognitive network doesn't have a license to function in the desired band. The fundamental elements of the primary and the unlicensed networks are defined as follow:

\section{a. Primary User:}

Primary user has a license to operate in a certain spectrum band. This access could be only controlled by its base-station and should not be affected by the operations of any other unauthorized user.

\section{b. Primary Base-Station:}

Primary base-station is a fixed infrastructure network component which has a spectrum license. The primary basestation does not have any cognitive radio capability for sharing spectrum with cognitive radio users. On the other hand, primary base-station may be required to have both legacy and cognitive radio protocols for the primary network access of cognitive radio users.

\section{c. Cognitive Radio User:}

Cognitive radio user has no spectrum license. Therefore, the spectrum access is allowed only in an opportunistic manner. Functions of the cognitive radio user include spectrum decision, spectrum sensing, spectrum handoff and cognitive radio MAC/routing/transport[9] protocols. The cognitive radio user is assumed to have the capabilities to communicate with not only the base-station but also other cognitive radio users.

\section{d. Cognitive Radio Base-Station:}

Cognitive radio base-station is a fixed infrastructure component with cognitive radio capabilities. CR base-station provides single hop connection to cognitive radio users without spectrum access license.

As shown in Figure 2, cognitive radio users can either communicate with each other in a multihop manner or access the base-station. As a result, in the cognitive radio network architecture, there are three different access ways over heterogeneous networks, which can show different implementation necessities as follows:

\section{a. Cognitive Radio Network Access:}

Cognitive radio users can access their own cognitive radio base-station both in licensed and unlicensed spectrum bands. While all interactions take place inside the cognitive radio network and their medium access design is independent of that of primary network.

\section{b. Cognitive Radio Ad Hoc Access:}

Cognitive radio users can communicate with other cognitive radio users through ad hoc connection on both licensed and unlicensed spectrum bands. Also the cognitive radio users may have their own medium access technology.

\section{c. Primary Network Access:}

The cognitive radio user can access the primary base-station through the licensed band if the primary network is allowable. Dissimilar other access types, cognitive radio users should support the medium access technology of primary network. In addition to primary basestation should support cognitive radio capabilities.

\section{d. Spectrum Sensing for Cognitive Radio Networks:}

A CR should monitor the available spectrum bands, capture the information, and then after detect the spectrum holes shows in fig 2 . Then, spectrum sensing is a key enabling technology in cognitive radio networks. In the spectrum sensing, the detection accuracy is considered as the most important factor to determine the performance of cognitive radio networks.

\section{e. Spectrum Efficiency:}

The main objective of cognitive radio is the efficient use of spectrum resources. Since CR's users can't not transmit during the sensing, spectrum efficiency would be degraded in evitable.

In Cognitive radio network, available spectrums may show different characteristics with the bandwidth, primary user activity and the acceptable interference limit, which influence both the sensing accurateness and spectrum efficiency. As a result, spectral proficient sensing technique[10] is essential for cognitive radio networks. Thus, in this project we will propose the spectral efficient sensing technique for cognitive radio networks, where it provides optimal spectrum sensing period and observation time to maximize the efficiency of each spectrum bands subject to the resource limitation and interference restriction.

\section{PROPOSED METHODOLOGY}

The wireless communication system has limited bandwidth with the high traffic with limited spectrum availability and to handle high load of data it is necessary to efficiently utilize the limited spectrum by using the sense the channel and switch the load to free or lower load channel. To make system intelligent the cognitive radio system provides the facilities to sense the spectrum[6] and detect any type of changes either noise, power or energy.

In Fig. 2 shows the block diagram of proposed system, where the main functional block are explained below. The preprocessing of signal is needed before transmission of signal. The transmitter block transmits the signal over wireless channel which is AWGN or Rayleigh Fading Channel. During transmission of channel the signal is encountered with the noises and the receiver end median filter reduce some noises before detection of signals. Here the use of median filter enhances the system performance significantly. 
Fig. 3.2 shows the flowchart of the proposed methodology where simulation starts with the initialization of environmental variables which creates the artificial model of practical cognitive radio system, after that the simulation threshold is defines for Monte-Carlo simulation now than a single tone signal is generated to sense the spectrum which than transmitted through channel but first there is need to initialize the channel.

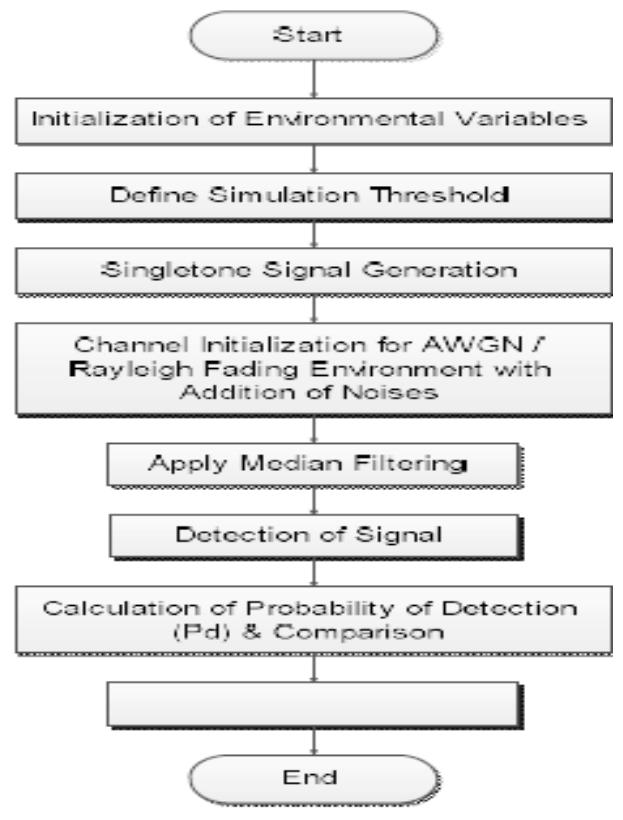

Fig. 2 Flowchart of proposed methodology

During transmission of noises will be added at the receiver which than reduced by median filter for efficient detection of spectrum sensing signal than finally signal is detected and the calculation of probability of detection $(\mathrm{Pd})$ is calculated for various values of signal to noise ratio (SNR) and the results are displayed.

\section{SIMULATION RESULTS}

The proposed system is explained in the precious section of this paper the system. In this section simulation is done under Monte-Carlo simulation method and the wireless channel considerations are AWGN channel and Rayleigh Fading Channel[1]. To increase the performance of the system median filter is employed at the detection end.

Fig. 3 to 6 shows the results of the system output in terms of probability if detection calculated for given values of signal to noise ratio (SNR) implementing with median filtering at detection end.

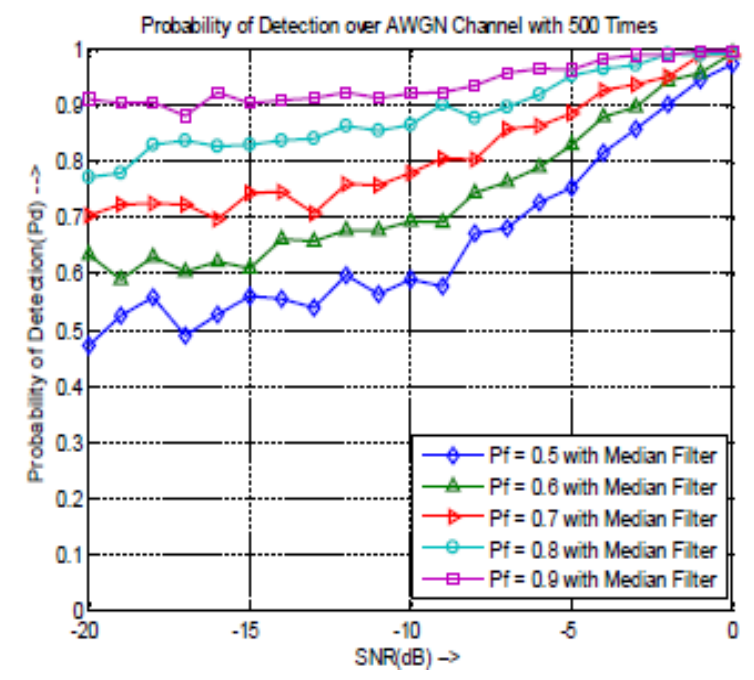

Fig. 3 Probability of Detection vs SNR for given false alarm rate with Median Filtering performing 500 iterations under AWGN Channel environment

The simulation results of proposed system as shown in Fig. 3 is performed under AWGN channel environment with 500 iterations and it is clear that the probability of detection is better for higher values of $\mathrm{Pf}$ (i.e. 0.9). But the results is better than the previous work for $\mathrm{Pf}=0.5$ and the value of it increases the values of Pd also increases. All the conditions of Pf giving optimum performance for lower values of signal to noise ration which is best outcome.

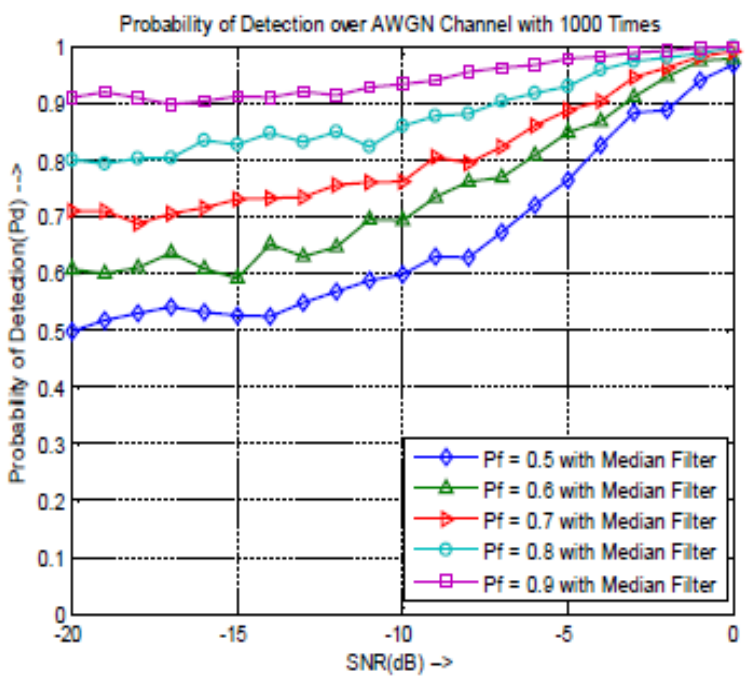

Fig. 4 Probability of Detection vs SNR for given false alarm rate with Median Filtering performing 1000 iterations under AWGN Channel environment 


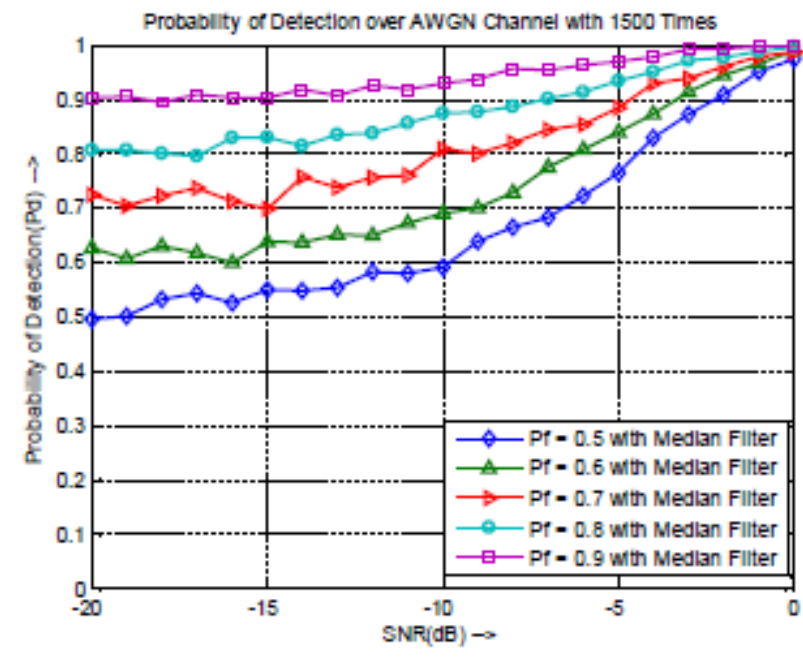

Fig. 5 Probability of Detection vs SNR for given false alarm rate with Median Filtering performing 1500 iterations under AWGN Channel environment

The simulation results of proposed system as shown in Fig. 4 is performed under AWGN channel environment with 1000 iterations and it is clear that the probability of detection is better for higher values of $\mathrm{Pf}$ (i.e. 0.9). But the results is better than the previous work for $\mathrm{Pf}=0.5$ and the value of it increases the values of Pd also increases. All the conditions of Pf giving optimum performance for lower values of signal to noise ration which is best outcome.

The simulation results of proposed system as shown in Fig. 5 is performed under AWGN channel environment with 1500 iterations and it is clear that the probability of detection is better for higher values of Pf (i.e. 0.9). But the results is better than the previous work for $\mathrm{Pf}=0.5$ and the value of it increases the values of Pd also increases. All the conditions of Pf giving optimum performance for lower values of signal to noise ration which is best outcome.

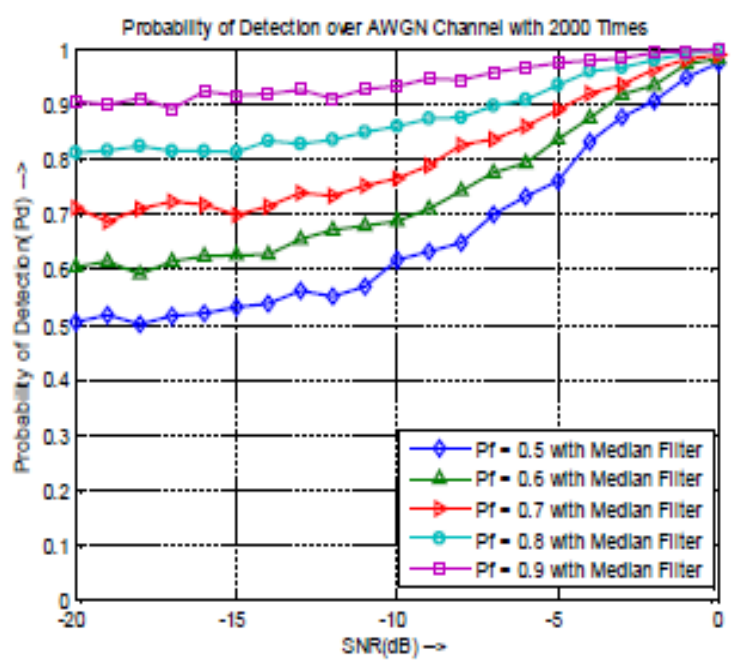

Fig. 6 Probability of Detection vs SNR for given false alarm rate with Median Filtering performing 2000 iterations under AWGN Channel environment

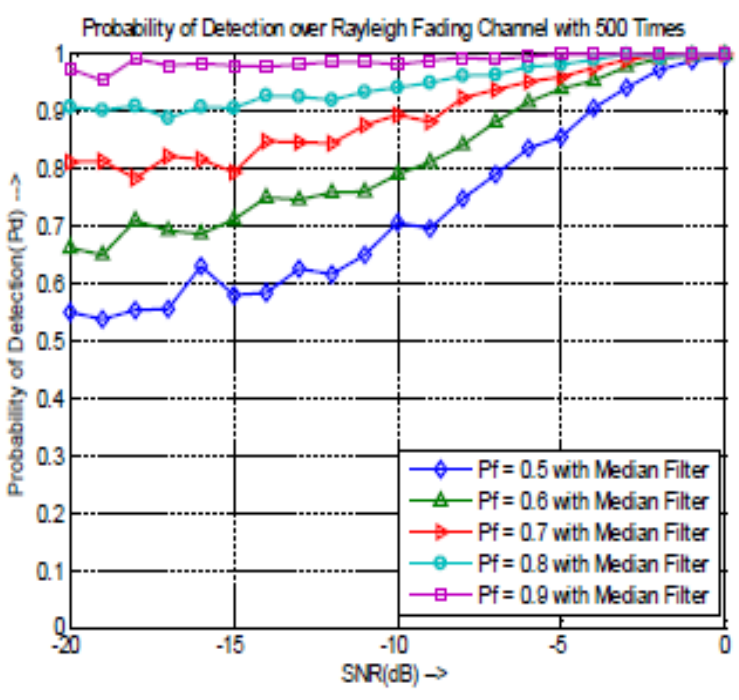

Fig. 7 Probability of Detection vs SNR for given false alarm rate with Median Filtering performing 500 iterations under Rayleigh Fading environment

The simulation results of proposed system as shown in Fig. 6 is performed under AWGN channel environment with 2000 iterations and it is clear that the probability of detection is better for higher values of $\mathrm{Pf}$ (i.e. 0.9). But the results is better than the previous work for $\mathrm{Pf}=0.5$ and the value of it increases the values of Pd also increases. All the conditions of Pf giving optimum performance for lower values of signal to noise ration which is best outcome.

The simulation results of proposed system as shown in Fig. 6 is performed under Rayleigh Fading channel environment with 500 iterations and it is clear that the probability of detection is better for higher values of $\mathrm{Pf}$ (i.e. 0.9). The results analyzed under Rayleigh Fading is better than AWGN Channel considerations. But the results is better than the previous work for $\mathrm{Pf}=0.5$ and the value of it increases the values of Pd also increases. All the conditions of Pf giving optimum performance for lower values of signal to noise ration which is best outcome.

The simulation results of proposed system as shown in Fig. 8 is performed under Rayleigh Fading channel environment with 1000 iterations and it is clear that the probability of detection is better for higher values of Pf (i.e. 0.9). The results analyzed under Rayleigh Fading is better than AWGN Channel considerations. But the results is better than the previous work for $\mathrm{Pf}=0.5$ and the value of it increases the values of Pd also increases. All the conditions of Pf giving optimum performance for lower values of signal to noise ration which is best outcome. 


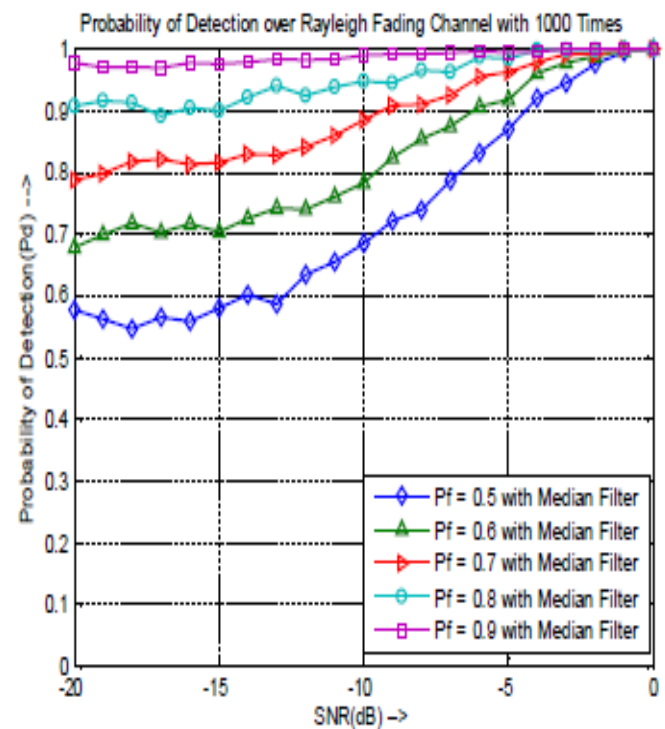

Fig. 9 Probability of Detection vs SNR for given false alarm rate with Median Filtering performing 1500 iterations under Rayleigh Fading environment

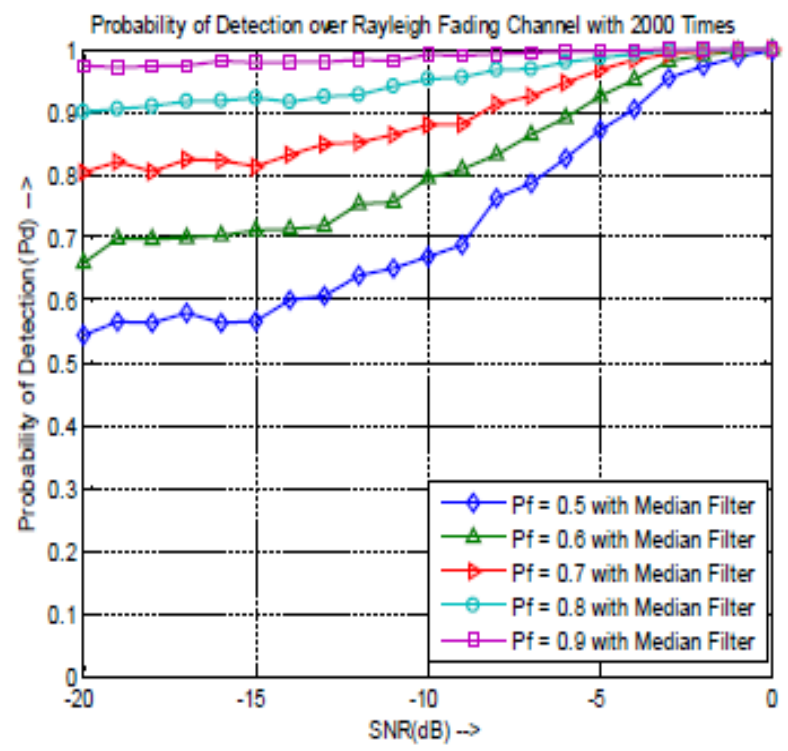

Fig. 10 Probability of Detection vs SNR for given false alarm rate with Median Filtering performing 2000 iterations under Rayleigh Fading environment

The simulation results of proposed system as shown in Fig. 6 is performed under Rayleigh Fading channel environment with 2000 iterations and it is clear that the probability of detection is better for higher values of Pf (i.e. 0.9). The results analyzed under Rayleigh Fading is better than AWGN Channel considerations. But the results is better than the previous work for $\mathrm{Pf}=0.5$ and the value of it increases the values of Pd also increases. All the conditions of Pf giving optimum performance for lower values of signal to noise ration which is best outcome.

\section{CONCLUSION AND FUTURE SCOPE}

From the simulation results it is realized that the proposed methodology using Monte-Carlo simulation and Median Filtering is better for intelligent cognitive radio networks and the system performance is better for lower values of signal to noise ratio i.e. $-20 \mathrm{~dB}$ which is as lower as possible. The false alarm rate should be significantly higher to keep the system intelligent as results says. In future the more complex and efficient filtering and simulation technique make system more intelligent and optimum for energy saving and utilization of bandwidth enhancing the data rates in the higher load condition for any one or two channels.

\section{REFERENCES}

[1] Tevfik Yucek and Huseyin Arslan, "A survey of spectrum sensing algorithms for cognitive radio applications," IEEE Comunications surveys \& tutorials, vol. 11, no. 1 , First Quarter 2015.

[2] "Notice of Proposed Rule Making and Order," FCC, ET Docket No. 03-322 December 2014.

[3] S. Haykin, "Cognitive Radio: Brain-Empowered Wireless Communications," IEEE Journal On Selected Areas in Communications, vol. 23, no. 2, pp. 201-220, 2013.

[4] Francisco Paisana, Neeli Prasad, Antonio Rodrigues, and Ramjee Prasad, "An alternativeimplementation of a cyclostationary detector," in The 15th International Symposium onWireless Personal Multimedia Communications, Taipei, Taiwan, 2012.

[5] J. Mitola, "Cognitive radio for flexible mobile multimedia communications," in IEEEInternational Workshop on Mobile Multimedia Communications, Nov. 2011, pp. 310.

[6] Y. Tachwali, F. Basma, and H. H. Refai, "Cognitive Radio Architecture for Rapidly Deployed Heterogeneous Wireless Networks," IEEE Transactions on Consumer Electronics, vol. 56, no. 3, pp. 1426-1432, 2010.

[7] -, "On the energy detection of unknown signals over fading channels," IEEE Trans.Commun., vol. 55, no. 1, pp. 21-24, Jan. 2009.

[8] Danijela Cabric, Artem Tkachenko, and Robert W. Brodersen, "Experimental Study ofSpectrum Sensing based on Energy Detection and Network Cooperation," in Proc. ACM Int. Workshop on Technology and Policy for Accessing, Boston, Aug. 2006.

[9] Amod V. Dandawate and Georgios B. Giannakis, "Statistical tests for presence of cyclostationarity," IEEE Transactions on Signal Processing, Sep. 1994.

[10] Wang Jun and Bi Guangguo, "Novel Autocorrelation Based Spectrum Sensing Methods for Cognitive Radios," in 16th Asia-Pacific Conference on Communications (APCC), 2010. 